\title{
LES DÉPÉRISSEMENTS LITTORAUX D'ARBRES FORESTIERS
}

\author{
J.-P. GARREC
}

Le littoral français est le plus étendu d'Europe avec $5500 \mathrm{~km}$ de côtes.

Le long de ce littoral, ies arbres rencontrent de nombreuses contraintes spécifiques et en particulier des contraintes climatiques (vent, embruns) et des contraintes stationnelles (sols filtrants et sécheresse édaphique, déséquilibres minéraux et salinité des sols).

Toutes ces contraintes sont le moteur d'une forte pression de sélection qui rend les arbres du littoral particulièrement sensibles lorsque des conditions climatiques exceptionnelles apparaissent (froid, tempête, sécheresse). Comme cela est observé en plusieurs endroits des côtes françaises, ces conditions extrêmes peuvent aussi contribuer à un affaiblissement général des arbres qui deviennent alors la cible des parasites et des maladies. À ces contraintes s'ajoutent maintenant l'action de polluants issus de sources qui peuvent être maritimes, mais également urbaines, agricoles et industrielles.

$\mathrm{Si}$, généralement, ces perturbations des arbres du littoral restent localisées, on constate que, lorsque plusieurs de ces contraintes se conjuguent (conditions stationnelles difticiles, pollution, salinité, vent), des phénomènes plus importants de dépérissement peuvent apparaître (Stammitti, 1992).

Actuellement, le long du littoral français, deux grandes zones sont concernées par des dépérissements de plus ou moins grandes envergures: la côte vendéenne et charentaise entre les embouchures de la Loire et de la Gironde, et la côte méditerranéenne, principalement dans les départements des Bouches-du-Rhône et du Var.

\section{LE DÉPÉRISSEMENT DU PIN MARITIME SUR LE LITTORAL NORD-ATLANTIQUE}

Ces forêts de Pin maritime du littoral nord-atlantique, où des phénomènes de dépérissement ont été observés, sont principalement situées entre l'île de Noirmoutier et celle d'Oléron, et sont installées essentiellement sur des dunes parallèles au littoral constituées de sables calcaires $(\mathrm{pH} \approx 9)$.

Cette zone de climat relativement homogène est caractérisée par une sécheresse au début de la période de végétation (printemps et début de l'été) et par des vents importants qui peuvent accélérer les phénomènes de sécheresse.

Ces forêts implantées au début du XIX siècle pour fixer les dunes sont fortement monospécifiques et comportent un mélange de variétés ibériques et aquitaines de Pin maritime (Pinus pinaster).

Des dépérissements localisés, qui se caractérisent par des jaunissements des aiguilles, ont régulièrement été observés depuis l'installation de ces forêts, mais une accélération du phénomène 
semble apparaître vers les années 1988. Sur les 6000 hectares de forêts littorales étudiées, $60 \%$ des peuplements ( 3500 hectares) sont touchés d'une façon discontinue par le dépérissement et, sur environ 1000 hectares, ce phénomène a pris des proportions inquiétantes (Guyon et Guyon, 1993).

Pour comprendre l'origine et l'évolution de ce dépérissement, un vaste programme de recherche a été initié à partir de 1988 par l'Office national des Forêts avec la collaboration de I'INRA de Bordeaux :

- mise en place d'un réseau de 23 placettes permanentes d'observations permettant de dresser des cartes du dépérissement ;

- utilisation de photographies aériennes en infrarouge couleur :

- mise en place d'arboretums de comportement ;

- mise en place, sur deux sites, d'un observatoire du fonctionnement des écosystèmes forestiers ;

- étude en laboratoire des sensibilités en $\mathrm{NaCl}$, au $\mathrm{pH}$ et au calcaire actif, des différentes variétés de pins maritimes;

- rôle des insectes et des champignons.

À partir des recherches déjà réalisées, les hypothèses suivantes se dégagent sur l'origine de ce dépérissement (Guyon, 1991):

- existence de facteurs prédisposants: un sol au pH très basique contenant de fortes concentrations en sodium ;

- rôle de facteurs déclenchants : relation avec de fortes pluies hivernales ou printanières ;

- présence de facteurs aggravants :

- l'origine génétique des pins : les pins landais sont plus résistants que les pins ibériques,

- l'emplacement des arbres : les plus sensibles sont situés en lisière de forêt, en bord de mer ou en zones dégagées,

- l'âge des arbres et la densité des peuplements.

Suite à ces études, des moyens de lutte à court et à long terme contre ce dépérissement et des nouvelles règles de gestion ont été mis en place:

- fertilisation des peuplements ;

- éclaircies sanitaires rapides;

— introduction de nouvelles espèces comme des feuillus ;

— amélioration des techniques d'introduction des jeunes plants : rideaux brise-vent.

En 1992, un léger répit a été constaté dans le jaunissement.

\section{LE DÉPÉRISSEMENT DES ARBRES SUR LE LITTORAL MÉDITERRANÉEN}

Depuis les années 1950, des dépérissements d'arbres sont apparus le long des côtes méditerranéennes, d'abord dans la région de Marseille pour s'étendre par la suite au cours des années, et d'une manière discontinue, sur toute la façade maritime ; on signale ainsi des dépérissements dans les départements de l'Hérault, de l'Aude, des Pyrénées-Orientales, des Alpes-Maritimes et du Var. Actuellement, les zones les plus touchées semblent être, outre la région Sud-Sud-Ouest de Marseille, la rade et les îles d'Hyères (Port-Cros et Porquerolles).

Ce dépérissement touche toutes les espèces d'arbres et se caractérise par des nécroses foliaires et par la chute des feuilles ou des aiguilles. D'une manière générale, les résineux avec leurs aiguilles 
persistantes sont les plus sensibles. Les tout premiers travaux ont rapidement permis de lier ce phénomène de dépérissement aux effets des embruns qui transportent un ou plusieurs composants phytotoxiques comme le fuel et les détergents (Devèze et Sigoillot, 1978).

Pour comprendre les causes et les mécanismes de ce dépérissement, plusieurs programmes de recherches ont été lançés. D'abord, des travaux ont été effectués par le Parc national de Port-Cros avec la collaboration de différents laboratoires des Universités d'Aix-Marseille pour connaître exactement l'étendue du phénomène sur la végétation littorale et son intensité. Par la suite, le ministère de l'Environnement dans le cadre du GIS "Posidonie " (1978), en collaboration avec les Universités de Marseille et de Toulon, a initié un ensemble de recherches sur:

- les caractéristiques physiques, chimiques et microbiologiques des embruns pollués ;

- les processus d'accumulation des polluants dans la microcouche de la surface de la mer;

- le suivi phytosociologique de la végétation côtière.

En 1992, I'INRA de Nancy, en collaboration avec les Universités de Marseille et de Besançon, a commencé un nouveau programme de recherche sur les mécanismes de la phytotoxicité des tensio-actifs vis-à-vis des surfaces foliaires et des cuticules, et sur les perturbations hydriques dans les feuilles (Garrec et Sigoillot, 1992).

Ces différents travaux ont confirmé le rôle phytotoxique des embruns qui atteignent la végétation, embruns pollués par des tensio-actifs (détergents provenant des rejets d'eaux usées d'origines industrielles ou ménagères) et par du fuel (provenant du trafic maritime). Ces recherches ont montré que :

- les rivages les plus touchés sont les rivages aux côtes peu accidentées ou avec des baies largement ouvertes ;

- les arbres atteints sont les arbres de première ligne, et les feuilles directement tournées vers la mer sont d'abord détruites ;

- les tensio-actifs agissent sur la perméabilité des feuilles en altérant les cuticules (dégradation des cires), et favorisent la pénétration du $\mathrm{NaCl}$ des embruns dans les tissus foliaires ;

- c'est l'accumulation du $\mathrm{NaCl}$ dans les feuilles qui est la principale responsable des nécroses et des dépérissements observés ;

- au niveau de la mer, les polluants s'accumulent dans les "slicks", zones présentant une surface unie et qui apparaissent par vent faible ;

- les concentrations de détergents et de fuel sont beaucoup plus importantes dans les embruns que dans l'eau de mer.

Ce dépérissement des arbres le long du littoral méditerranéen est indéniablement d'origine anthropique et est lié dans cette région à la pollution du milieu marin suite au développement du trafic maritime et à la présence de grands centres industrialo-portuaires. Par la suite, cette pollution maritime se transforme en pollution de l'air.

Les moyens de lutte contre ce dépérissement sont actuellement limités et passent avant tout par une diminution du niveau de pollution de la mer.

\section{CONCLUSIONS}

Ces deux dépérissements des arbres du littoral français ont des origines totalement diftérentes.

Le dépérissement des arbres du littoral atlantique est avant tout la conséquence de conditions climatiques particulières (fortes pluies d'hiver et de printemps), de conditions stationnelles difficiles 
(sols très basiques et riches en sodium) auxquelles se rajoutent des facteurs aggravants liés à l'origine. l'âge et la position des arbres.

Par contre, le dépérissement des arbres du littoral méditerranéen est le résultat d'une pollution de la mer par des détergents et des hydrocarbures dont les embruns pollués transportés par les vents violents atteignent les arbres. Pour l'instant, ce dépérissement ne se rencontre en Europe que le long des côtes méditerranéennes, mais il atteint par exemple des proportions beaucoup plus importantes en Italie dans les environs de l'embouchure de l'Arno.

Ces deux importants dépérissements, présents actuellement le long des côtes françaises, mettent en évidence l'importance de conditions climatiques exceptionnelles et/ou de la pollution comme facteurs de déclenchement de graves perturbations dans ces écosystèmes forestiers fragiles.

On peut alors se poser des questions sur l'avenir de ces arbres du bord de mer. En effet, dans le cadre des futurs changements climatiques et des conséquences de l'effet de serre, les différents scénarios considèrent comme probable, dans les zones littorales, une augmentation des vents, une hausse du niveau de la mer et de fortes irrégularités des précipitations. Parallèlement, il est prévu un développement accéléré de l'urbanisation et de l'implantation d'industries dans ces zones, avec tous les problèmes de pollution que cela va poser.

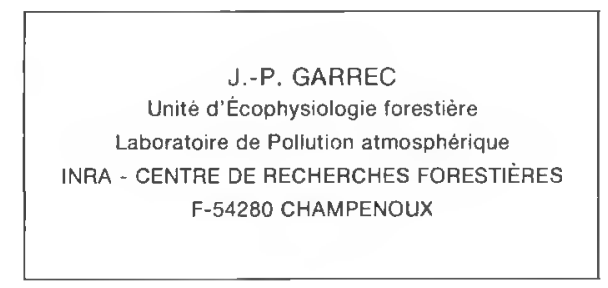

\section{BIBLIOGRAPHIE}

DEVĖZE (L.), SIGOILLOT (J.-C.). - Les Arbres malades de la mer. - Eau et aménagement de la région provençale, $n^{\circ} 19,1978$, pp. 13-24.

GARREC (J.-P.), SIGOILLOT (J.-C.). - Les Arbres malades de la mer. - La Recherche, vol. 23, $n^{\circ} 245,1992$, pp. $940-941$.

GIS-POSIDONIE. - Programme Embruns marins polluės. Rapport final. — Contrat SRETIE/MERE/89017, 1990. $80 \mathrm{p}$.

GUYON (J.-P.). - Dépérissement du Pin maritime (Pinus pinaster Ait) en Vendée. Les causes écologiques. Annales des Sciences forestières, vol. 48, 1991, pp. 333-346.

GUYON (J.-P.), GUYON (D.). - Le Dépérissement du Pin maritime sur le littoral nord-atlantique. Résultats des recherches 1988-1992, - Office national des Forêts - Département des Recherches techniques - Interrégion "Ouest", 1993. - 31 p.

STAMMITTI (L.). - Le Dépérissement de la végétation en bordure du littoral. — Les Éditions du STU - Ministère de I'Équipement, 1992. - $131 \mathrm{p}$. 